\title{
Action video game training reduces the Simon Effect
}

\author{
Claire V. Hutchinson ${ }^{1} \cdot$ Doug J. K. Barrett ${ }^{1} \cdot$ Aleksander Nitka $^{1} \cdot$ Kerry Raynes $^{1}$
}

Published online: 4 August 2015

(C) Psychonomic Society, Inc. 2015

\begin{abstract}
A number of studies have shown that training on action video games improves various aspects of visual cognition including selective attention and inhibitory control. Here, we demonstrate that action video game play can also reduce the Simon Effect, and, hence, may have the potential to improve response selection during the planning and execution of goal-directed action. Non-game-players were randomly assigned to one of four groups; two trained on a first-personshooter game (Call of Duty) on either Microsoft Xbox or Nintendo DS, one trained on a visual training game for Nintendo DS, and a control group who received no training. Response times were used to contrast performance before and after training on a behavioral assay designed to manipulate stimulus-response compatibility (the Simon Task). The results revealed significantly faster response times and a reduced cost of stimulus-response incompatibility in the groups trained on the first-person-shooter game. No benefit of training was observed in the control group or the group trained on the visual training game. These findings are consistent with previous evidence that action game play elicits plastic changes in the neural circuits that serve attentional control, and suggest training may facilitate goal-directed action by improving players' ability to resolve conflict during response selection and execution.
\end{abstract}

Research Highlights - Performance on the Simon Task was assessed before and after video game training

- Training on a first-person-shooter game improved task performance

- Findings may reflect enhanced conflict resolution during response selection and execution

Claire V. Hutchinson

ch190@le.ac.uk

1 School of Psychology, College of Medicine, Biological Sciences and Psychology, University of Leicester, Leicester LE1 9HN, UK
Keywords Video game training $\cdot$ Reaction times $\cdot$ Response selection · Simon Effect

\section{Introduction}

In recent years, a body of literature has emerged concerning the positive effects of action video game training on perception and cognition. Compared to non-game players, action video game players (i.e., people who play first-person shooter games) have lower contrast-detection thresholds (Li, Polat, Makous, \& Bavelier, 2009) and are more sensitive to contracting optic flow (Hutchinson \& Stocks, 2013). Video game play has also been associated with an increase in the speed of visual processing and faster response times (RTs) (Dye, Green, \& Bavelier, 2009). Game play decreases the attentional blink (Green \& Bavelier, 2003), increases search efficiency (Wu \& Spence, 2013), and decreases attentional capture by task-irrelevant distractors (Chisholm, Hickey, Thweeuwes, \& Kingstone, 2010). These improvements have been observed in non-game players who have undergone as little as 10 hours' training, indicating rapid changes in perceptual sensitivity and attentional control that generalize from game play to laboratory and real-world tasks (see Bavelier, Green, Pouget, and Schrater (2012) and Spence and Feng (2010) for reviews).

Action video games are characterized by highly complex, dynamic displays. Game play requires the identification and selection of relevant visual information, followed by motor response selection and execution. In many respects, this is similar to the demands of everyday life, but with the important distinction that in fast-paced, immersive action video game environments, the execution of an appropriate motor response to the appearance of a relevant stimulus must be much more rapid than is typically required in the real world. A key 
question in this regard is how video game training affects each stage of the visuo-motor decision process. Many of the benefits associated with action game play have been attributed to enhanced top-down attentional control during the identification and selection of task-relevant stimuli (Cain, Prinzmetal, Shimamura, \& Landau, 2014; Green \& Bavelier, 2003; Wu \& Spence, 2013). Game play modulates late event-related potential (ERP) components thought to reflect the endogenous modulation of stimulus-driven competition for selection (Wu, Cheng, Feng, D’Angelo, Alain, \& Spence, 2012), and has been associated with changes in the activation of a frontoparietal network implicated in executive control and the dynamic allocation of cognitive resources (Bavelier, Green, Pouget, \& Schrater, 2012; Kuhn, Lorenz, Banaschski, et al., 2014). Few studies, however, have directly examined the effects of action game play on motor response and selection.

West and colleagues (2013) compared oculomotor control in non-game and game players. They found game players exhibited larger saccadic deviations away from distractors when they were required to fixate a target object during search. These results provide initial evidence that improved attentional control in game players generalizes from target identification to response selection and visuo-motor control. To investigate this possibility further, we used the Simon Task to assess whether action game play improves response selection and inhibition for manual responses. The Simon Task presents one of two possible targets on each trial. Targets differ on a non-spatial feature such as their color, and each target is arbitrarily assigned a left- or right-hand response. Observers make a speeded-response using the appropriate hand to identify the target, which can appear in the left or right hemifield. When the target and response are spatially incongruent (i.e., the target assigned a right-hand response appears in the left hemifield), RTs are typically slower than when the target and response are spatially congruent. Because the location of the target on each trial is irrelevant to the response, the magnitude of the RT difference is considered a measure of the observer's ability to select the appropriate action when the target and the response elicit competing spatial representations (Forstmann, van den Wildenberg, \& Ridderinkhof, 2008; Simon \& Rudell, 1967).

Although the precise mechanisms underlying the Simon Effect remain controversial (see Hommel, 2011, for a review), a number of models emphasize the role of attentional control in the suppression of irrelevant spatial information during response selection (Eimer, 1999; Ridderinkhof, van den Wildenberg, Wijnen, \& Burle, 2002). In behavioral studies, the resolution of response conflict has been attributed to the dynamic weighting of response tendencies during the planning and execution of visuo-motor control (De Jong, Liang, \& Lauber, 1994; Forstmann et al., 2008a, b). Neuroimaging data implicate a network of neural regions in this process, with the inferior and dorsolateral prefrontal cortices
(DLPFC) and the anterior cingulate cortex associated with the endogenous weighting of task-relevant information and response selection, respectively (Liu, Banich, Jacobson, \& Tanabe, 2004; Wyllie, Ridderinkhof, Elias, et al., 2010). Recent evidence has also revealed an association between habitual game play and the cortical thickening of left DLPFC (Kuhn, Lorenz, Banaschski, et al., 2014), an area associated with attentional control during target detection and response selection. If action video game play elicits plastic changes in the processes associated with the latter, practice would be expected to reduce the Simon Effect. Alternatively, if benefits in the endogenous control of stimulus-driven competition are restricted to target identification, action game play might be expected to produce an overall decrease in RTs that is independent of the spatial relationship between the visual target and the required response.

\section{Material and methods}

Sixty participants with no prior experience of video game play were recruited to the study. All had normal or corrected-tonormal visual acuity, and no history of eye disease. All experimental methods adhered to the tenets of the Declaration of Helsinki. The School of Psychology's Ethics Committee at the University of Leicester approved the study and informed consent was obtained from all participants before testing.

Each participant performed two sessions of the Simon Task, with 10 days in-between. Following the first test session, participants were randomly assigned to one of three training groups: (1) Call of Duty: Modern Warfare 3 for Microsoft Xbox $(n=15)$; (2) Call of Duty: Modern Warfare 3 for Nintendo DS ( $\mathrm{n}=15)$; and (3) Sight Training for Nintendo DS $(n=15)$. Each participant played the game to which they were assigned for 1 hour per day over ten sessions. After training, they returned for a second testing session, during which they repeated the Simon Task. The second testing session took place the day after training, rather than immediately after the last training session. This reduced the potential confound of arousal effects in the second testing session. A separate group of 15 participants was assigned to a no-training (control) group. These participants completed the two sessions of the Simon Task with no training in-between.

Participants received course credit for their participation. Those assigned to one of the training groups received an additional cash payment on completion of the study. There was some attrition during training. By the second test session, 14 participants remained in the Call of Duty for Microsoft Xbox group (12 females, two males), ten remained in the Call of Duty for Nintendo DS group (nine females, one male), and ten remained in the Sight Training for Nintendo DS groups (nine females, one male). In the no-training group, only 11 attended the second test session (eight females, three males). A number 
of participants complained of boredom, indicating this was the most likely reason for attrition. However, this was not the case in those training on the Xbox, which provided a much more immersive gaming environment. This highlights the importance of considering how engaging a computer-based training regime needs to be to provide an effective game-based therapy. Comfort during game-play could also potentially be an issue, in that the Xbox controller may be more comfortable to use than the controls on the Nintendo DS.

A standard Simon Task was used. At the viewing distance of $60 \mathrm{~cm}$, the display subtended $25^{\circ}$ vertically and $34^{\circ}$ horizontally. Participants were presented with a central fixation cross, after which they initiated the onset of the target with a key press (spacebar). Targets $\left(1.34 \times 1.34^{\circ}\right)$ were either a red or a green square, presented on a black background, $8.2^{\circ}$ to the left or right of central fixation (midpoint of the target). Prior to the main testing session, participants underwent a practice session of 12 trials to ensure they understood the task and could discriminate the targets.

In the test proper, 160 targets were presented. Fifty percent of the targets were red squares and $50 \%$ green squares. For a given target color, the probability that it would appear to the left or right of midline was equal (see Fig. 1). The participants' task was to identify the color of the target as quickly as possible by pressing a key with their right or left hand to report red and green targets, respectively. After they responded to a target, the fixation cross re-appeared and the participant initiated the next trial. Targets remained on the screen until participants responded, or for a maximum of 2,000 milliseconds (ms). If the response took less than $200 \mathrm{~ms}$, timed-out, or was incorrect, the trial was discarded and resampled in the sequence.

\section{Results}

Performance in the first (pre-training) test session confirmed the suitability of the experimental paradigm for producing the Simon Effect. Participants were significantly slower to respond to the presence of spatially incongruent targets than to spatially congruent targets $[t(59)=9.10, p<.0001]$. In the context of the present study, this means they were quicker to respond to a red target presented on the right and a green target presented on the left than to a red target presented on the left and a green target presented on the right (Fig. 1).

Figure 2 shows mean RTs (ms) for spatially congruent and incongruent targets on the first (before training) and second (after training) test sessions. Training groups $(\mathrm{a}-\mathrm{c})$ and the notraining group (d) are shown. Overall, RTs were faster on spatially congruent than incongruent trials $[F(1,41)=97.00$, $\left.p<.05, \eta_{p}{ }^{2}=0.70\right]$, demonstrating a robust Simon Effect. RTs were also faster on the second compared to the first test session $\left[F(1,41)=23.30, p<.05, \eta_{p}{ }^{2}=.362\right]$, and the Test session by Congruence interaction was significant $[F(3,41)=7.02, p$
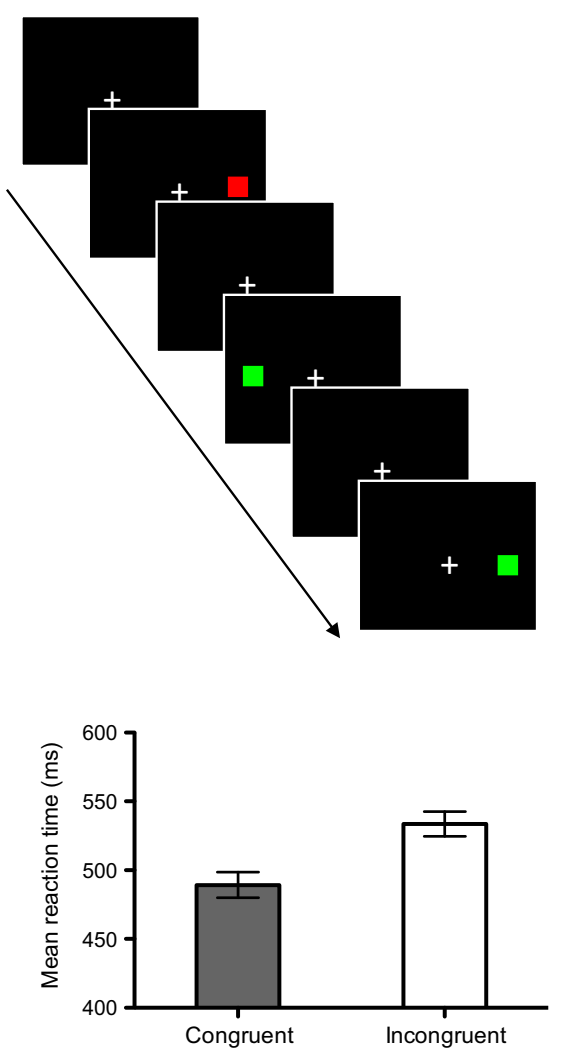

Fig. 1 Top: Example of the Simon Effect Task. Participants were presented with a red or a green square. Their task was to respond with their right hand upon presentation of a red square and their left hand upon presentation of a green square. Red and green squares appeared to the right or left of fixation, with equal probability. Bottom: Pre-training reaction times for responding to congruent (same side for presentation and response) or incongruent (different side for presentation and response) in the original 60 participants recruited to the study. Error bars are \pm 1 SEM

$\left.<.05, \eta_{p}{ }^{2}=.146\right]$, indicating a general reduction in RTs with training and a larger effect on incongruent compared to congruent trials. There was no overall effect of group $[F(3,41)=$ $\left..366, p>.05, \eta_{p}{ }^{2}=.026\right]$ nor was there a Congruence by Group interaction $\left[F(3,41)=.453, p>.05, \eta_{p}{ }^{2}=.032\right]$, Test session by Group $\left[F(3,41)=2.154, p>.05, \eta_{p}{ }^{2}=.136\right]$, and three-way Congruence by Test session by Group $[F(3,41)=$ $\left.1.197, p>.05, \eta_{p}{ }^{2}=.081\right]$ interactions were not significant. A comparison of the numbers of trials resampled for each participant revealed equivalent performance across experimental groups $\left[F(3,41)=.509, p>.05, \eta_{p}{ }^{2}=.036\right]$ in both sessions $\left[F(3,41)=.236, p>.05, \eta_{p}{ }^{2}=.054\right]$, ruling out speedaccuracy trade-offs as an explanation of differences in RTs.

Figure 2 reveals a larger mean decrease in RTs on incongruent (IC) than congruent (CG) trials in the second compared to the first session in the groups trained on Call of Duty. To explore this effect, we calculated individual changes in the Simon Effect $\left(\mathrm{SE}=\mathrm{RT}_{\mathrm{IG}}-\mathrm{RT}_{\mathrm{CG}}\right)$ at session 2 as a percentage of those in Session 1 [ $\left.\left(\mathrm{SE}_{2}-\mathrm{SE}_{1}\right) / \mathrm{SE} 1 * 100\right]$. Unlike the ANOVA above, this comparison excludes inter-subject 
(a) Call of Duty: XBox

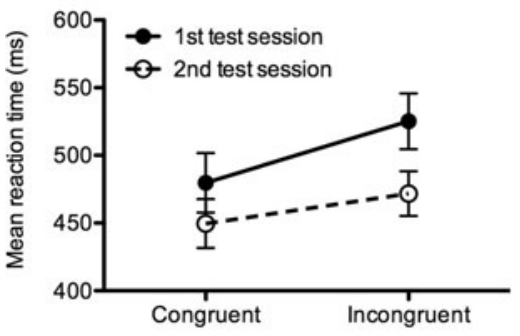

(c) Sight Training: Nintendo DS

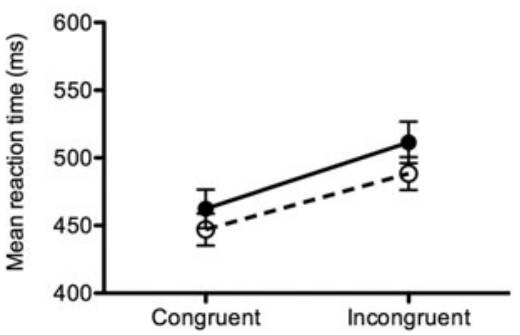

Fig. 2 Mean reaction times (ms) for congruent and incongruent targets for each training group: (a) Call of Duty for the XBox, (b) Call of Duty for the Nintendo DS, (c) Sight Training for the Nintendo DS. Performance is shown before (closed symbols) and after (open

variability in RTs that is not specific to the difference between incongruent and congruent trials. Individual changes in the Simon Effect were then subject to planned comparisons for each group ( $\mathrm{SE}_{2}<\mathrm{SE}_{1} ; 1$-sample $t$-tests; $\left.p=0.05\right)$. Training on Call of Duty reduced the Simon Effect for 10/14 and 9/10 of participants in the Xbox and Nintendo DS groups, producing significant mean decreases of $-49.86 \%[t(13)=1.94, p=$ $<.05]$ and $-64.71 \%[t(9)=2.79, p<.05]$, respectively. Decreases in the Simon Effect on the second compared to the first session were also observed for $5 / 10$ and $8 / 11$ of participants in the Sight Training and No-training control groups. Despite this, the mean Simon Effect for both groups was slightly higher in the second compared to the first session, producing non-significant increases of $10.42 \%[t(9)=0.29, p>0.05]$ and $8.29 \%[t(10)=0.18, p>0.05]$, respectively (see Fig. 3). The results, therefore, reveal a reliable reduction in the Simon Effect between sessions that was specific to participants trained on Call of Duty for Xbox and Nintendo DS.

\section{Discussion}

In the present study, we found a modest effect of action video game training on response selection and execution using the Simon Test. The results reveal a reliable reduction in RTs after 10 days of training and a decrease in the size of the Simon Effect among participants trained on Call of Duty for both the Xbox and Nintendo DS. These findings add to evidence that action game play can reduce RTs on a number of laboratory (b) Call of Duty: Nintendo DS

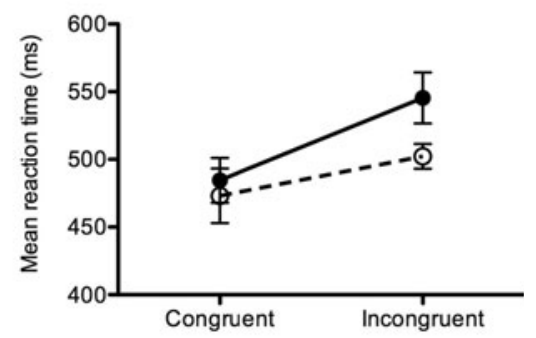

(d) No training

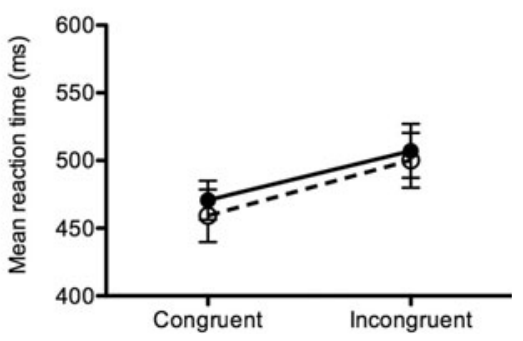

symbols) 10 hours of training. (d) Mean reaction times for no-training (control) group, who performed the task twice, but received no video game training. Error bars are \pm 1 SEM

tasks (Dye et al., 2009). The absence of a reduction in the Simon Effect for participants in the Sight Training or control groups is also consistent with previous evidence for training improvements that are specific to action games (Wu \& Spence, 2013). In addition, our results provide the first direct evidence of training that generalizes from action game play to a laboratory task used to induce conflict during response selection (Hommel, 2011). This finding supports the prediction that action game play can improve players' ability to reduce interference between competing response tendencies in order to facilitate goal-directed action. This interpretation is easily reconciled with evidence from studies that have employed the

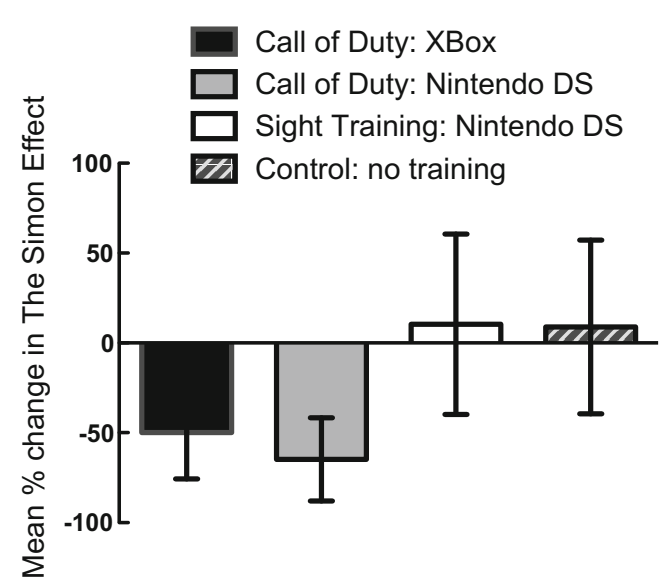

Fig. 3 Mean percentage change in the Simon Effect after training for each group. Negative values represent a reduction in the Simon Effect between the first and second sessions. Error bars are \pm 1 SEM 
Flanker task to show action game players are able to exclude information at irrelevant spatial locations better than nongame players (e.g., Durlach, Kring, \& Bowens, 2009; Green $\&$ Bavelier, 2003, 2007). Furthermore, our data extend these findings, and suggest action play can improve players' ability to inhibit irrelevant spatial information during the planning and execution of motor responses (De Jong, Liang, \& Lauber, 1994; Forstmann et al., 2008a, b).

The reduction of the Simon Effect among the Call of Duty groups in this study is statistically reliable but small. This is likely to reflect the limited sample size, as well as variability in the magnitude and direction of the change across participants. Despite this, the observed reduction provides support for a causal link between action game play and improved cognitive control during response selection and execution. This finding adds to a literature characterized by two approaches: Some studies have compared groups of individuals assigned as "action video gamers" and "non-video gamers" while others, including the present study, trained groups of non-video game players on video games. These different approaches raise interesting issues about how to interpret the results. One issue concerns why people play action video games in the first place. Individuals who play action video games do so for the rewards associated with achieving their goal (e.g., completing a given level and progressing to the next). It makes sense, therefore, that individuals who already possess good visuospatial skills are likely to play action video games. This makes it difficult to establish the direction of causality when associations are found. Studies that sample non-video game players who are subsequently "trained," provide a measure of the direct causal effects of action video game play on performance. These studies, however, are a much greater undertaking and are vulnerable to attrition (as in the present study). Selecting non-game players is also likely to introduce sample biases, as females and older adults are much less likely to play action video games than males between the ages of 20 and 35 years (Flurry Analytics, 2013). For example, in our study, the students we trained were mostly females $(84 \%)$ between the ages of 18 and 25 years. In this context, an important direction for future research would be to investigate training-related changes relative to pre-training visuo-spatial ability among samples that match the demographics of the general population, or the clinical subgroups most likely to benefit from game-based therapy.

We found that the Simon Effect was reduced after Call of Duty training in both Xbox and DS gaming formats. This raises issues concerning the precise characteristics of the game that improve perceptual performance and response selection. The interface of the Xbox and the DS are fundamentally different: The Xbox provides a largefield, immersive environment, while game play on the DS is less realistic due, principally, to the inferior graphics (relative to the Xbox) and restricted field of view. Despite this, both formats require the dynamic deployment of attention in order to select relevant information and guide appropriate responses. In this context, the benefits associated with action game play are consistent with a reversed hierarchy of perceptual learning where training improves perceptual sensitivity and motor control via feedback connections from higher cortical regions that mediate general cognitive control (Fahle, 2005).

The findings reported here are likely to have implications in a number of therapeutic contexts, including age-related decline in vision, attention, and cognition, and in rehabilitation after stroke. Action game play has also been linked to improved performance in professions requiring accurate perceptual discrimination and motor control (Sclickum, Hedman, Enochsson, et al., 2009), while commercially developed "brain training" software and active game environments have the potential to improve cognitive flexibility and response selection in aging (Walinsky, Unverzagt, Smith et al., 2006) and clinical populations (Li, Ngo, Nguyen, \& Levi, 2011). The effectiveness of game play training, however, is likely to reflect the format and tempo of the game as well as differences between players. Our data revealed a large degree of inter-subject variability in the effect of action game training on the Simon Effect. This variability has been noted in previous studies (Ridderinkhof et al., 2002), and may be related to individual differences in the structure and plasticity of the neural circuits associated with attentional control, response inhibition, and the acquisition of cognitive flexibility during game play (Forstmann, van den Wildenberg, \& Ridderinkhof, 2008b; Forstmann, Jahfari, Scholte, et al., 2008; Erikson, Boot, Basak, et al., 2010). Despite this, the emergence of a reliable reduction in the Simon Effect amongst non-gamers after only 10 hours' training illustrates the general potential of action game play to improve peoples' ability to allocate cognitive resources in order to optimize the selection and execution of motor responses.

Acknowledgments This work was supported by a British Academy grant to $\mathrm{CVH}$. CVH is grateful to the University of Leicester for a period of academic study leave.

\section{References}

Bavelier, D., Green, C. S., Pouget, A., \& Schrater, P. (2012). Brain plasticity through the life span: Learning to learn and action video games. Annual Review of Neuroscience, 35, 391-416.

Cain, M. S., Prinzmetal, W., Shimamura, A. P., \& Landau, A. N. (2014). Improved control of exogenous attention in action video game players. Fpsyg, 5, 69.

Chisholm, J. D., Hickey, C., Thweeuwes, J., \& Kingstone, A. (2010). Reduced attentional capture in action video game players. Attention, Perception, \& Psychophysics, 72, 667-671.

De Jong, R., Liang, C. C., \& Lauber, E. (1994). Conditional and unconditional automaticity: A dual process model of effects of stimulus- 
response correspondence. Journal of Experimental Psychology: Human, 20, 731-750.

Durlach, P. J., Kring, J. P., \& Bowens, L. D. (2009). Effects of action video game experience on change detection. Military Psychology, $21,24-39$.

Dye, M. G. W., Green, C. S., \& Bavelier, D. (2009). Increasing speed of processing with action video games. Current Directions in Psychological Science, 18, 321-326.

Eimer, M. (1999). Facilitory and inhibitory effects of masked prime stimuli on motor activation and behavioral performance. Acta Psychologica, 101, 293-314.

Erikson, K. I., Boot, W. R., Basak, C., Neider, M. B., Prakash, R. S., Voss, M. W., ... Kramer, A. F. (2010). Striatal volume predicts level of video game skill acquisition. Cerebral Cortex, 20, 2522-2530.

Fahle, M. (2005). Perceptual learning: Specificity versus generalization. Current Opinion in Neurobiology, 15, 154-160.

Flurry Analytics (2013). Top 200 iOS games tracked by Flurry [Blog post]. Retrieved from http://blog.apptopia.com/gamedemographics-that-every-developer-should-know/

Forstmann, B. U., Jahfari, S., Scholte, H. S., Wolfensteller, U., van den Wildenberg, W. P. M., \& Ridderinkhof, K. R. (2008a). Function and structure of the right inferior cortex predict individual differences in response inhibition: A model-based approach. The Journal of Neuroscience, 28, 9790-9796.

Forstmann, B., van den Wildenberg, W. P. M., \& Ridderinkhof, R. (2008b). Neural mechanisms, temporal dynamics, and individual differences in interference control. Journal of Cognitive Neuroscience, 20, 1845-1865.

Green, C. S., \& Bavelier, D. (2003). Action games modify visual selective attention. Nature, 423, 534-537.

Green, C. S., \& Bavelier, D. (2007). Action-video-game experience alters the spatial resolution of vision. Psychological Science, 18, 88-94.

Hommel, B. (2011). The Simon effect as a tool or heuristic. Acta Psychologica, 136, 189-202.

Hutchinson, C. V., \& Stocks, R. (2013). Selectively enhanced motion perception in core video gamers. Perception, 42, 675-677.

Kühn, S., Lorenz, R., Banaschewski, T., Barker, G. J., Büchel, C., Conrod, P. J., ... Gallinat, J. (2014). Positive Association of Video Game Playing with Left Frontal Cortical Thickness in Adolescents. PLOS ONE, 9, e91506.

Li, R., Polat, U., Makous, W., \& Bavelier, D. (2009). Enhancing the contrast sensitivity function through video game training. Nature Neuroscience, 12, 549-551.
Li, R. W., Ngo, C., Nguyen, J., \& Levi, D. M. (2011). Video game play induces plasticity in the visual system of adults with amblyopia. PLoS Biol, 9. doi:10.1371/journal.pbio. 1001135

Liu, X., Banich, M. T., Jacobson, B. L., \& Tanabe, J. L. (2004). Common and distinct neural substrates of attentional control in integrated Simon and spatial Stroop task as assessed by event-related fMRI. NeuroImage, 22, 1097-1106.

Ridderinkhof, K. R., van den Wildenberg, W. P. M., Wijnen, J., \& Burle, B. (2002). Response inhibition in conflict tasks is revealed in delta plots. In Posner (Ed.), Attention. New York: Guildford Press.

Sclickum, M. K., Hedman, L., Enochsson, L., Kjellin, A., \& FellanderTsai. (2009). Systematic video game training in surgical novices improves performance in virtual reality endoscopic surgical simulators: A prospective randomized study. World Journal of Surgery, 33, 2360-2367.

Simon, J. R., \& Rudell, A. P. (1967). Auditory S-R compatibility: The effect of an irrelevant cue on information processing. The Journal of Applid Psychology, 51, 300-304.

Spence, I., \& Feng, J. (2010). Video games and spatial cognition. Review of General Psychology, 14, 92-104.

Walinsky, F. D., Unverzagt, F. W., Smith, D., Jones, R., Stodard, A., \& Tennstedt, S. L. (2006). The active cognitive training trial and health-related quality of life: Protection that lasts for 5 years. The Journals of Gerontology. Series A, Biological Sciences and Medical Sciences, 61, 1324-1329.

West, G. L., Al-Aidroos, N., \& Pratt, J. (2013). Action video game experience affects oculomotor performance. Acta Psychologica, 142, $38-42$.

Wu, S., \& Spence, I. (2013). Playing shooter and driving video games improves top-down guidance in visual search. Attention, Perception, \& Psychophysics, 74, 673-686.

Wu, S., Cheng, C. K., Feng, J., D’Angelo, L., Alain, C., \& Spence, I. (2012). Playing a first-person shooter video game induces neuroplastic change. Journal of Cognitive Neuroscience, 24, 1286-1293.

Wyllie, S. A., Ridderinkhof, K. R., Elias, W. J., Frysinger, R. C., Bashmore, T. R., Downs, K. E., ... van den Wildenberg, W. P. (2010). Subthalamic nucleus stimulation influences expression and suppression of impulsive behaviour in Parkinson's disease. Brain, 133, 3611-3624. 